MATEC Web of Conferences 37, 01024 (2015)

DOI: $10.1051 /$ matecconf/20153701024

C) Owned by the authors, published by EDP Sciences, 2015

\title{
ANALYTICAL ESTIMATION OF THE CENTRAL REFLECTOR IMPACT ON THERMAL NEUTRON FLUX DENSITY IN RESEARCH REACTORS
}

\author{
Dmitry V. Gvozdyakov" a, Anton O. Tanishev ${ }^{1}$, Svetlana A. Shvab ${ }^{1}$, Vladimir N. Martyshev ${ }^{1}$, Arian V. \\ Kuzmin ${ }^{1}$ \\ ${ }^{1}$ Institute of Power Engineering, National Research Tomsk Polytechnic University, Lenina Avenue, 30, 634050, \\ Tomsk, Russia
}

\begin{abstract}
In this research was shown an analytical assessment of the impact of the central reflector as an example of spherical reactor in one-group approximation. From the external reflector introduced an effective border that simplifies the mathematical problem of critical task. The validation of the research is done just for one of the IRT-T reactor conditions.
\end{abstract}

\section{Mathematical model and study technique}

In the academic literature assessments of the impact of the central reflector is not given [1-3]. In some works a crucial problem was solved for planar geometry of the one-group, one-group modified [4] and in the diffusion-age approximation [6-8].

It should be noted [1-2], that the inner central reflectors are used in the projects of research and power reactors to increase the thermal neutron flux and for the energy alignment on the core of a nuclear reactor. These results can be widely applied in practice, e.g. in solving the acute problem of central reflector masonry beryllium poisoning, (on the example of the IRT-T research reactor).

The purpose of this research is to solve the critical problem as an example of a homogeneous spherical reactor with internal and external reflectors in the single-group approximation. To simplify the problem, is used the method of effective boundary conditions, following which from the outside of the reflector introduce efficient frontier R. Graphical interpretation is shown in Figure 1.

a Corresponding author : Dim2003@tpu.ru

This is an Open Access article distributed under the terms of the Creative Commons Attribution License 4.0, which permits unrestricted use, distribution, and reproduction in any medium, provided the original work is properly cited. 

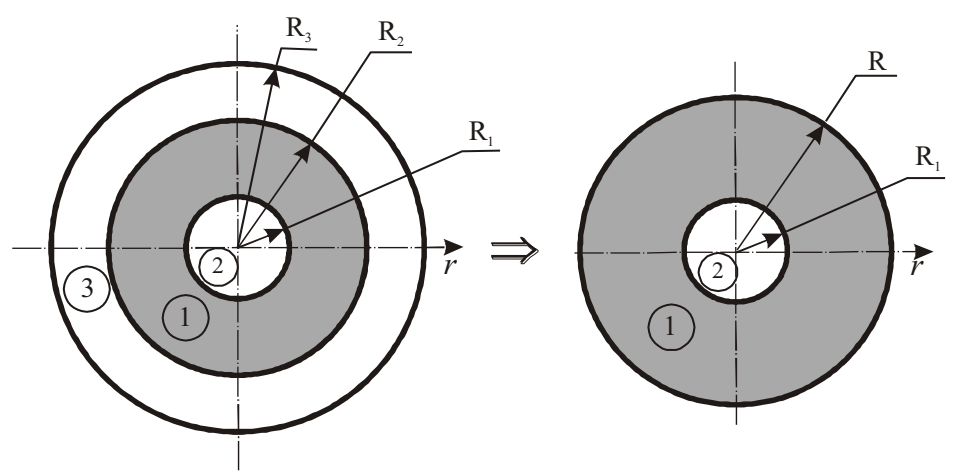

Figure 1. The transformation of the initial problem with the implementation of the efficient boundary: 1-active zone; 2 internal reflector; 3 external reflector

The distribution of the neutron flux in the core, the inner and outer reflectors in the one-group approximation is usually described by the following equations:

$$
\begin{gathered}
\frac{\mathrm{d}^{2} \Phi_{1}(\mathrm{r})}{\mathrm{dr}^{2}}+\frac{2}{\mathrm{r}} \frac{\mathrm{d} \Phi_{1}(\mathrm{r})}{\mathrm{dr}}+\chi_{1}^{2} \cdot \Phi_{1}(\mathrm{r})=0, \\
\frac{\mathrm{d}^{2} \Phi_{2}(\mathrm{r})}{\mathrm{dr}^{2}}+\frac{2}{\mathrm{r}} \frac{\mathrm{d} \Phi_{2}(\mathrm{r})}{\mathrm{dr}}-\chi_{2}^{2} \cdot \Phi_{2}(\mathrm{r})=0,
\end{gathered}
$$

where $\chi_{1}, \chi_{2}-$ material parameters of the media core and reflectors respectively.

Using the following conditions we will find the equations solution (1), (2):

$$
\begin{gathered}
1^{\mathrm{o}} \cdot r=0 ; 0<\Phi_{2}(0)<\infty, \\
2^{\mathrm{o}} \cdot r=\mathrm{R}_{1} ; \Phi_{2}\left(\mathrm{R}_{1}\right)=\Phi_{1}\left(\mathrm{R}_{1}\right), \\
3^{\mathrm{o}} . r=\mathrm{R}_{1} ; \mathrm{D}_{2} \nabla \Phi_{2}\left(\mathrm{R}_{1}\right)=\mathrm{D}_{1} \nabla \Phi_{1}\left(\mathrm{R}_{1}\right), \\
4^{\mathrm{o}} \cdot r=\mathrm{R}=\mathrm{R}_{2}+\delta_{\mathrm{ef}} ; \Phi_{1}(\mathrm{R})=0,
\end{gathered}
$$

where $D_{i}$ is diffusivity.

The use of an external reflector reduces neutron leakage and saves the core is equal to the effective additive [2].

Assume that a reasonable border of the outer reflector is less than $2 \cdot \mathrm{L}_{3}$ (because there is onegroup evaluation and leak will be through this border). Wave equations solutions (1) and (2) are of the form:

- for the core (in the shape of a sphere): 


$$
\Phi_{1}(r)=C_{1} \cdot \frac{\sin \left(\chi_{1} \cdot r\right)}{r}+C_{2} \cdot \frac{\cos \left(\chi_{1} \cdot r\right)}{r},
$$

- for the reflector (in the form of a sphere):

$$
\Phi_{2}(r)=C_{3} \cdot \frac{\operatorname{sh}\left(\chi_{2} \cdot r\right)}{r}+C_{4} \cdot \frac{\operatorname{ch}\left(\chi_{2} \cdot r\right)}{r} .
$$

To obtain the distribution of the neutron flux explicitly there was introduced an additional boundary condition $\mathrm{r}=\mathrm{R}_{1}$, assuming that we know the instrumental power of the nuclear reactor and the corresponding neutron flux density at the boundary of the inner core and the reflector.

Figure 2 is a graphical solution to the above mentioned problem.

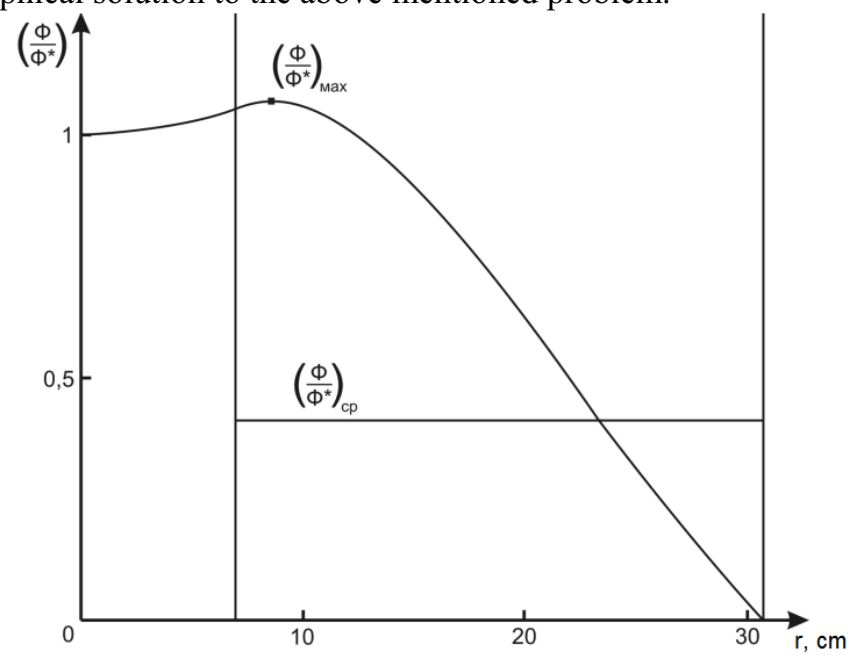

Figure. 2. The distribution of the neutron flux for the reactor core and the inner reflector

Thus, reflectors efficiency can be evaluated by the average thermal neutron flux ratio change in the core to its maximum value.

For the reactor without reflector this value is equal to 0,304 [3]. If we have a central reflector - it will be 0,384 in interval $\left[R_{1}, R\right]$ and 0,531 for reactor with two reflectors in $\left[R_{1}, R_{2}\right]$.

Although one-group model doesn't take into account the cumulative effect of the thermal neutrons due to the moderation of the fast neutrons in reflectors, in general terms it represents their effectiveness in the reactor energy alignment.

In terms of neutron physics we consider thermal neutron flux. It was found out that a high density of thermal neutron flux could improve the radiopharmaceuticals quality.

In conclusion, there should be noted that the goal of this research has been achieved. There was found an analytical solution distribution of thermal neutron flux in one-group approximation for spherical reactor with a central reflector. A feature of the analytical assessment is that the external reflector was replaced with the effective border (for simplification), but it's possible to use internal reflector in order to increase the density distribution of the neutron flux in reflector or in reactor's active zone.

The work has been done in FCAEI HE "National Research Tomsk Polytechnic University" in framework of federal focused program implementation "Research and Development on Priority 


\section{MATEC Web of Conferences}

Orientation of Science and Technology Complex Development in Russia for 2014-2020”, unique identifier 'Applied Research and Experimental Developments' RFMEFI58114X0001.

\section{References}

1. V.A. Varlachev, O.F. Gusarov. IRT-T research nuclear reactor: Manuals manufacturing practice and probation (TPU, Tomsk, 2002)

2. G.G. Bartholomew, G.A. Bat, V.D. Baybakov, M.S. Alkhutov. Fundamentals of the theory and methods of nuclear power reactors calculation: tutorial for high school (Energoatomizdat, Moscow, 1989).

3. R. Murray, Nuclear reactors physics, (1961)

4. Tsyplenkov K.V., A.V. Kuzmin. Modern technique and technologies: Proceedings of young scientists in XI International Scientific Conference, 2, (2005).

5. D.V. Gvozdyakov, R.A. Tarasenko, A.V. Kuzmin. Energy: ecology, reliability, safety: Mater. 12 All-Russian Scientific Conference, (2006).

6. A.V. Kuzmin. News TPU, 1, (2007).

7. R.E. Vodyankin, D.V. Gvozdyakov, A.V. Kuzmin, Energy: ecology, reliability, safety: Mater. 14 All-Russian Scientific Conference, (2008). 